\title{
Distinct Presynaptic and Postsynaptic Dismantling Processes of Drosophila Neuromuscular Junctions during Metamorphosis
}

\author{
Zhiwei Liu, ${ }^{1,2}$ Yan Chen, ${ }^{1}$ Dan Wang, ${ }^{1}$ Songyan Wang, ${ }^{1}$ and Yong Q. Zhang ${ }^{1}$ \\ ${ }^{1}$ Key Laboratory for Molecular and Developmental Biology, Institute of Genetics and Developmental Biology, Chinese Academy of Sciences, Beijing 100101, \\ China, and ${ }^{2}$ College of Fisheries and Life Science, Shanghai Ocean University, Shanghai 201306, China
}

\begin{abstract}
Synapse remodeling is a widespread and fundamental process that underlies the formation of neuronal circuitry during development and in adaptation to physiological and/or environmental changes. However, the mechanisms of synapse remodeling are poorly understood. Synapses at the neuromuscular junction (NMJ) in Drosophila larvae undergo dramatic and extensive remodeling during metamorphosis to generate adult-specific synapses. To explore the molecular and cellular processes of synapse elimination, we performed confocal microscopy, live imaging, and electron microscopy (EM) of NMJ synapses during the early stages of metamorphosis in Drosophila in which the expressions of selected genes were genetically altered. We report that the localization of the postsynaptic scaffold protein Disc large (Dlg) becomes diffuse first and then undetectable, as larval muscles undergo histolysis, whereas presynaptic vesicles aggregate and are retrogradely transported along axons in synchrony with the formation of filopodia-like structures along NMJ elaborations and retraction of the presynaptic plasma membrane. EM revealed that the postsynaptic subsynaptic reticulum vacuolizes in the early stages of synapse dismantling concomitant with diffuse localization of Dlg. Ecdysone is the major hormone that drives metamorphosis. Blockade of the ecdysone signaling specifically in presynaptic neurons by expression of a dominant-negative form of ecdysone receptors delayed presynaptic but not postsynaptic dismantling. However, inhibition of ecdysone signaling, as well as ubiquitination pathway or apoptosis specifically in postsynaptic muscles, arrested both presynaptic and postsynaptic dismantling. These results demonstrate that presynaptic and postsynaptic dismantling takes place through different mechanisms and that the postsynaptic side plays an instructive role in synapse dismantling.
\end{abstract}

\section{Introduction}

Selective elimination of neuronal processes takes place widely in neuronal development and pathological conditions, and is crucial for the formation and plasticity of mature neural circuitry (Lichtman and Colman, 2000; Luo and O'Leary, 2005). Studies on synapse elimination of mammalian neuromuscular junction (NMJ) have contributed substantially to our understanding of the process. In mammals, multiple motor neurons initially send out axons that converge at the target muscles. Within the first few postnatal weeks, however, all but one of the NMJ synapses are eliminated (Colman et al., 1997; Lichtman and Colman, 2000). The elimination is not caused by neuronal death but by local pruning of axons and engulfment of axon tips by Schwann cells (Keller-Peck et al., 2001; Bishop et al., 2004). Postsynaptic factors

Received Jan. 24, 2010; revised July 8, 2010; accepted July 14, 2010.

This work was supported by National Natural Science Foundation of China Grants 30430250 and 30525015, and Ministry of Science and Technology of China Grant 2007CB947200 (Y.Q.Z.). We thank V. Budnik, K. Ito, D. W. Williams, W. M. Saxton, S. Stowers, H. D. Ryoo, R. K. Murphey, and Hugo Bellen for stocks and antibodies; Xiaoping Chen and Chuanmao Zhang for assistance in EM analyses; and F. Huang, X. Huang, M. Ding, A. Boulanger, and J. Dura for discussions and comments on this manuscript.

Correspondence should be addressed to Dr. Yong Q. Zhang, Institute of Genetics and Developmental Biology, Chinese Academy of Sciences, No. 1 Datun Road, Chao Yang District, Beijing 100101, China. E-mail: yqzhang@genetics.ac.cn.

DOI:10.1523/JNEUROSCI.0410-10.2010

Copyright $\odot 2010$ the authors $\quad 0270-6474 / 10 / 3011624-11 \$ 15.00 / 0$ and neuronal activity have been found to be required for synapse elimination in the peripheral NMJ and the central cerebellum, respectively (McCann et al., 2007; Lorenzetto et al., 2009).

Widespread remodeling of neuronal projections happens during Drosophila metamorphosis to accommodate the switch from larva to adult physiology and behavior. This involves largescale selective elimination and rewiring of neural circuits. Thus, Drosophila metamorphosis has been widely used to investigate the mechanisms underlying the elimination of neuronal processes. Most insights into neuronal remodeling come from studies on axonal pruning of mushroom body neurons in the brain (Lee et al., 2000; Zheng et al., 2003; Awasaki and Ito, 2004; Watts et al., 2004; Awasaki et al., 2006) and dendritic pruning of peripheral sensory neurons (Kuo et al., 2005, 2006; Williams and Truman, 2005; Williams et al., 2006; Lee et al., 2009). So far, ecdysone signaling, TGF- $\beta$ signaling, and the ubiquitinproteasome system have been shown to regulate neuronal pruning (Lee et al., 2000; Watts et al., 2003; Zheng et al., 2003; Kuo et al., 2006). In addition to the molecular pathways, cellular processes such as glial engulfment and phagocytosis by blood cells have been found to participate in the process (Awasaki and Ito, 2004; Watts et al., 2004; Williams and Truman, 2005; Awasaki et al., 2006). All these studies have focused on the elimination of neuronal processes. But how synapses are eliminated in Drosophila is unclear. 
We therefore sought to characterize the changes taking place during synapse elimination and to identify the pathways involved in the process. To this end, we performed confocal microscopy, live imaging, and electron microscopy of NMJ synapses in wildtype and mutant Drosophila during metamorphosis. Our results reveal previously undocumented mechanisms of presynaptic and postsynaptic elimination. Inactivation of the ecdysone signaling in presynaptic neurons delayed presynaptic but not postsynaptic dismantling. However, inhibiting histolysis of the postsynaptic muscles by tissue-specific blockade of ecdysone signaling, ubiquitination pathway, or apoptosis arrested both presynaptic and postsynaptic dismantling, indicating the dependence of presynaptic dismantling on postsynaptic dismantling, but not vice versa. Our data together demonstrate that the postsynaptic side plays an instructive role in synapse elimination. Synapse elimination at the Drosophila NMJ during metamorphosis affords a paradigm for dissecting the molecular pathways that govern the process.

\section{Materials and Methods}

Fly stocks and culture. All flies were raised on standard media at $25^{\circ} \mathrm{C}$ unless otherwise specified. Strain Oregon R was used as the wild-type control. The transgenic Syt-eGFP elav-Gal4/FM7 that labels presynaptic vesicles in all neurons was described previously (Zhang et al., 2002). MHC-CD8-GFP-Shaker that labels the postsynaptic membrane (Zito et al., 1999) and the muscle-specific MHC-Gal4 were from C. Goodman, University of California, Berkeley, CA. Another stronger muscle-specific Gal4 line BG57-Gal4 was from V. Budnik, University of Massachusetts, Worcester, MA (Budnik et al., 1996). A motor neuron-specific OK6-Gal4 was from M. O'Connor, University of Minnesota, Minneapolis, MN. SNAP-25-YFP that labels presynaptic plasma membrane was provided by S. Stowers, Montana State University, Bozeman, MT. Transgenic flies carrying UAS-GFP, UAS-shits and glia-specific Repo-Gal4 were from K. Ito, University of Tokyo, Tokyo, Japan. Transgenic flies carrying insertions encoding dominant-negative mutations of ecdysone receptors (EcR) EcR-A-F645A (referred to as EcR.A ${ }^{\mathrm{ND}}$ ) and EcR-B1- $\Delta 655$-F645A (referred to as EcR.B1 ${ }^{\mathrm{ND}}$ ) were from L. Cherbas, Indiana University, Bloomington, IN (Cherbas et al., 2003). UAS-Ubp2 was from A. DiAntonio, Washington University School of Medicine, St. Louis, MO (DiAntonio et al., 2001). UAS-P35 was from the Bloomington Stock Center. UAS-Diap1 was from H. Ryoo, New York University School of Medicine, New York, NY. UAS-glued ${ }^{1}$ was from R. Murphey, University of Massachusetts, Amherst, MA (Allen et al., 1999). Dhc64C $C^{4-19}$ and Dhc64C $C^{6-10}$ were from W. Saxton, University of California, Santa Cruz, CA (Martin et al., 1999).

Pupal staging and dissection. White prepupae [0 $\mathrm{h}$ after puparium formation (APF)] were collected and placed on a coverglass in a Petri dish with moist filter paper as previously described (Bainbridge and Bownes, 1981). All cultures were raised at $25 \pm 0.5^{\circ} \mathrm{C}$ and $60 \pm 5 \%$ relative humidity. Pupae before $7 \mathrm{~h}$ APF were cut open along the dorsal midline and dissected in PBST (PBS with $0.3 \%$ Triton-X), followed by fixation in $4 \%$ paraformaldehyde in PBS for 30 min before immunostaining. Pupae after $7 \mathrm{~h}$ APF were fixed in 4\% paraformaldehyde in PBS for 15 min before dissection followed by an additional $15 \mathrm{~min}$ of fixation.

Immunohistochemistry. Immunostaining of larval and pupal samples was performed primarily as previously described (Zhang et al., 2001; Jin et al., 2009). Briefly, fixed larval and pupal preparations were washed in PBST three times (each $10 \mathrm{~min}$ ), blocked in blocking solution (PBST with $5 \%$ normal goat serum) for $30 \mathrm{~min}$, and incubated in primary antibody for $2 \mathrm{~h}$ at room temperature or overnight at $4^{\circ} \mathrm{C}$. The following primary antibodies were used: Texas Red-X or FITC-labeled goat anti-HRP (1:50; The Jackson Laboratory), rabbit anti-synaptotagmin (1:1000; gift from H. Bellen, Baylor College of Medicine, Houston, TX), rabbit antiglutamate receptor (GluR) IIB (1:1000; gift from A. DiAntonio), mouse anti-Disc large (Dlg) (1:1000; 4F3), anti-Bruchpilot (1:40; NC82), and anti-GluR IIA (1:50; 8B4D2); the latter three were from the Developmental Studies Hybridoma Bank. Different fluorophore-conjugated second- ary antibodies against rabbit or mouse IgG were from Sigma-Aldrich and used at 1:1000 in PBST. All images were collected using a Leica SP5 laser-scanning confocal microscope and processed with Adobe Photoshop 8.0.

NMJ quantifications were performed primarily based on published procedures (Jin et al., 2009). All images analyzed were projections from complete $z$-stacks through the entire NMJ 4 of abdominal segment A3 or A4. The number of filopodial structures (see Fig. 2) was manually scored based on anti-HRP staining. Bouton number, size, and synaptic area of third-instar larvae and pupae at different developmental stages were statistically analyzed according to anti-synaptotagmin staining. NIH ImageJ 3.0 was used to define the anti-synaptotagmin-stained boutons and report the area of each bouton or total boutons automatically. The area of total boutons in pupae at different stages was normalized to that of the corresponding larvae (see Figs. 2, 5, 7). All statistical comparisons were performed using GraphPad InStat 5 software. Values of $p$ were calculated by two-tailed Student's $t$ test.

Time-lapse imaging of synapse dismantling. Animals aged from thirdinstar larvae to pupae $0-10 \mathrm{~h}$ APF were dissected in $\mathrm{Ca}^{2+}$-free HL3 solution (Stewart et al., 1994). The spread-out neuromusculature was then mounted and immersed in modified HL3 solution with a $\mathrm{Ca}^{2+}$ concentration of $1.0 \mathrm{~mm}$. Time-lapse images of synaptic vesicles (SVs) labeled by Syt-eGFP driven by the pan-neuronal elav-Gal4 or presynaptic neuronal membrane labeled by SNAP25-YFP driven by the motor neuron specific OK6-Gal4 from abdominal segments 3 or 4 were captured with an Olympus microscope via water-immersion lens every 10-30 min at $20^{\circ} \mathrm{C}$.

Electron microscopy. Larvae and early pupae for electron microscopy (EM) were prepared according to the procedures described previously (Zito et al., 1999). Dissected larval and pupal neuromusculatures were fixed for $2 \mathrm{~h}$ with $2.5 \%$ glutaraldehyde (Sigma-Aldrich) in cacodylate buffer, $\mathrm{pH} 7.4$, at room temperature followed by several rinses with cacodylate buffer. Left and right hemisegments from abdominal segments 3 and 4 were separated from the larval or pupal fillets and postfixed with $1 \% \mathrm{OsO}_{4}$ in cacodylate buffer for $2 \mathrm{~h}$. The preparations were stained en bloc for $1 \mathrm{~h}$ with saturated uranyl acetate in $50 \%$ ethanol before dehydration in graded ethanol. The tissue was embedded in Spurr resin (Sigma-Aldrich), and ultrathin sections were made on an LKB ultramicrotome or with a Leica UC6 using a diamond knife. Grids were poststained with saturated uranyl acetate in $50 \%$ ethanol and $1 \%$ lead citrate, pH 12, and examined with a JEOL 1010 electron microscope. Micrographs were taken with a Ganton 792 digital CCD.

\section{Results}

\section{Elimination of NMJ synapses in the early stages of metamorphosis}

Drosophila NMJ synapses have been widely used to study synaptic mechanisms for over 30 years since the classic work of Jan and Jan (1976) but have rarely been used to study synapse elimination. In the present study, we have used NMJ synapses in the early stages of metamorphosis as a model system in which to dissect the molecular and cellular process of synapse elimination. Most, if not all, larval motor neurons innervating the abdominal muscles remodel during metamorphosis to innervate adult abdominal muscles (Tissot and Stocker, 2000). To obtain an overview of synapse elimination during metamorphosis, transgenic flies with NMJ synapses labeled with MHC-CD8-GFP-Shaker (Zito et al., 1999) were used. CD8-GFP-Shaker is a chimeric protein containing the extracellular and transmembrane domains of the human T-lymphocyte protein CD8, the green fluorescent protein (GFP), and the cytoplasmic C-terminal sequence of the potassium channel Shaker. When the chimeric CD8-GFP-Shaker is driven by the myosin heavy chain (MHC) promoter, it expresses in all muscles and labels the postsynaptic densities of type $1 \mathrm{~b}$ (big) and type $1 \mathrm{~s}$ (small) boutons via its interaction with the postsynaptic scaffold protein Dlg. However, type $1 \mathrm{~b}$ boutons are brightly labeled, whereas type $1 \mathrm{~s}$ boutons are only weakly labeled, because type $1 \mathrm{~b}$ 

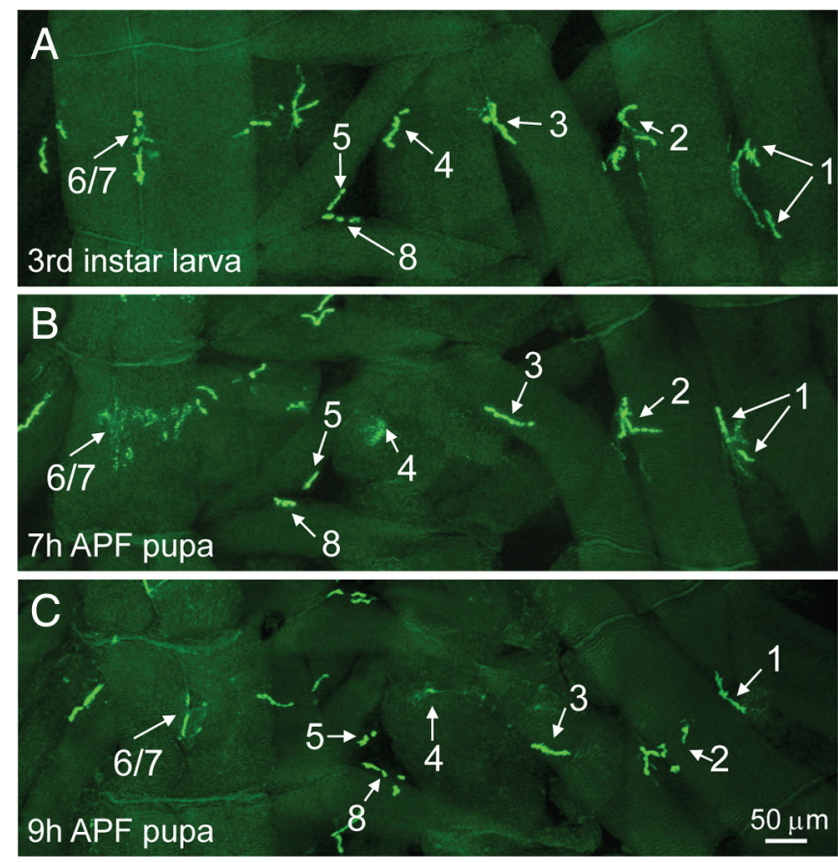

Figure 1. Overview of NMJ synapse elimination during the early stages of metamorphosis. NMJs were labeled by a transgenic GFP fusion protein CD8-GFP-Shaker driven by MHC promoter in postsynaptic muscles. $A-C$, Representative NMJ images of internal layers of muscles in abdominal segment A3 in a third-instar larva (A), $7 \mathrm{~h}$ APF pupa (B), and $9 \mathrm{~h}$ APF pupa (C). NMJs 1-8 are indicated. GFP signals of NMJs 4 and $6 / 7$ were diffuse at $7 \mathrm{~h} \mathrm{APF} \mathrm{(B).} \mathrm{At} 9 \mathrm{~h} \mathrm{APF,} \mathrm{NMJ} \mathrm{6/7}$ was greatly reduced, whereas NMJ 4 had almost completely disappeared (C). NMJs 1,2,3,5, and 8 in the $9 \mathrm{~h}$ APF pupa $(\boldsymbol{C})$ showed no obvious changes compared with their larval counterparts (A). Scale bar, $50 \mu \mathrm{m}$.

synapses have a more elaborate subsynaptic reticulum (SSR) in which CD8-GFP-Shaker is localized (Zito et al., 1999).

As shown in Figure $1 A$, different muscles of third-instar larvae have distinctive NMJ synapse elaborations. At $7 \mathrm{~h}$ APF, NMJ synapses labeled with CD8-GFP-Shaker at muscles 4 and 6/7 of the abdominal segment A3 were diffuse, whereas NMJ synapses on muscles $1,2,3,5$, and 8 resembled their larval counterparts (Fig. $1 B$ ). At 9 h APF, labeling of NMJ 4 had almost completely disappeared, whereas labeling of NMJ 6/7 was greatly reduced. However, NMJs 1, 2, 3, 5, and 8 of the segment A3 remain unaltered compared with their larval equivalents (Fig. 1C). These data suggest that NMJ synapses in different muscles of the same abdominal segment are eliminated at different times. We also observed that NMJ 4 of the abdominal segment A2 remained intact at $7 \mathrm{~h} \mathrm{APF}$ and was still present at $9 \mathrm{~h} \mathrm{APF} \mathrm{(data} \mathrm{not}$ shown), whereas the NMJ 4 of segment A3 disappeared at $9 \mathrm{~h}$ APF (Fig. 1C), indicating that NMJ synapses on the same muscle at different abdominal segments are eliminated at different stages. In general, NMJ synapses in the posterior abdominal segments were eliminated earlier than those in the anterior segments.

Postsynaptic Dlg becomes diffuse before presynaptic vesicles aggregate and the presynaptic membrane retracts during synapse elimination

The dramatic dismantling of NMJ synapses in a narrow time window during early metamorphosis (Fig. 1) is well suited for studying the mechanisms of synapse elimination. Given the asynchronous elimination of different NMJs in different muscles of different abdominal segments, we chose the type $1 \mathrm{~b}$ NMJ of muscle 4 in A3 or A4 segment to study synapse elimination for the following reasons.
Table 1. Timetable for the elimination of various presynaptic and postsynaptic components

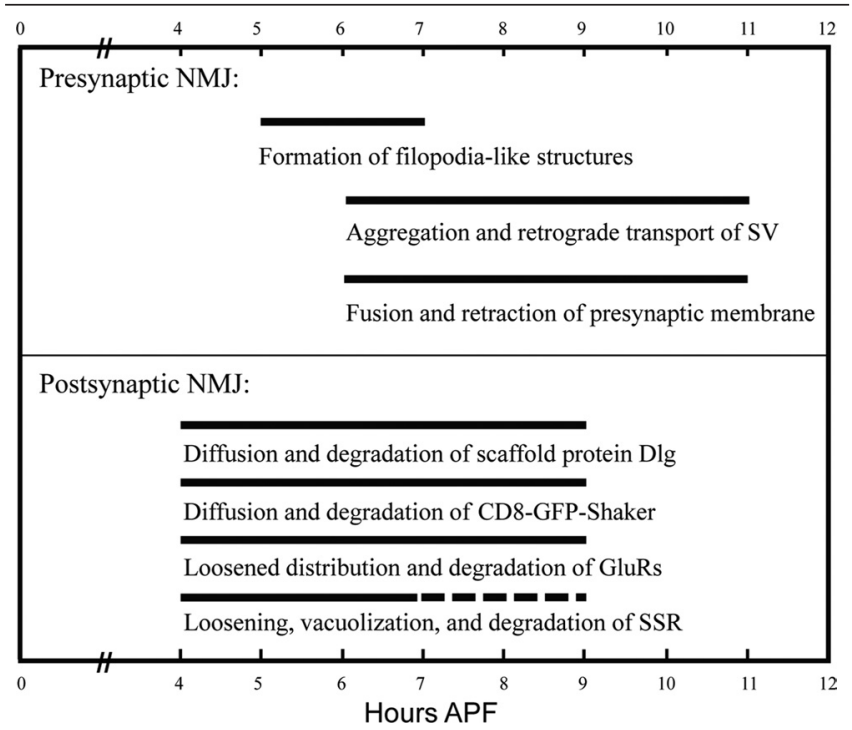

Postsynaptic elimination starts $1 \mathrm{~h}$ before presynaptic elimination. Presynaptic filopodial structures were observed at 5-7 h APF. Active zone component Bruchpilot is disassembled concomitantly with GluRs. Loosening and vacuolization of SSR were observed at $4-7 \mathrm{~h}$ APF by EM. The gapped line indicates disassembly of SSR, which has not been experimentally demonstrated.

First, muscle 4 is large and easy to identify during metamorphosis because it lies between persistent muscle 1 and muscle 8 . Second, muscle 4 is innervated by only two motor neurons, MN4a with type $1 \mathrm{~b}$ boutons and MN4b with type 1s boutons (Wan et al., 2000). Moreover, the type $1 \mathrm{~b}$ NMJ, referred to as NMJ 4 in this study, is located at the center of muscle 4 not obscured by neighboring NMJs and has a simpler elaboration pattern than those in other muscles such as NMJ 6/7. Third, NMJ 4 of abdominal segments A3 or A4 is dismantled synchronously and completely by $\sim 11 \mathrm{~h}$ APF (Fig. 1, Table 1), thus allowing visualization of the complete synapse dismantling process in a short period of time.

To analyze the process of synapse elimination in detail, we immunostained NMJ synapses with different synaptic markers in third-instar larvae and early pupae at 6, 7, and 9 h APF (Fig. 2). The morphology of NMJ synapses changes dramatically during the metamorphic period we examined (Fig. 2). In a third-instar larva, NMJ 4 labeled by different synaptic markers showed a typical "beads-on-a-string" pattern of synaptic boutons (Fig. 2A1A4). The staining pattern of presynaptic membrane detected by anti-HRP mostly overlapped with that of SVs labeled by an antibody against synaptotagmin (Syt), a transmembrane protein of SV. At $6 \mathrm{~h}$ APF, Dlg staining was diffuse (Fig. 2B1), whereas prominent presynaptic filopodial structures were observed by anti-HRP staining (Fig. 2B3). Some of the filopodial structures contained Syt-positive signals (Fig. 2, compare B3, B2). At $7 \mathrm{~h}$ APF, Dlg staining became more diffuse (Fig. 2C1) and the number of filopodial structures decreased dramatically from $7.31 \pm$ 0.73 (mean \pm SEM) in $6 \mathrm{~h} \mathrm{APF}$ pupae to $2.53 \pm 0.47$ in $7 \mathrm{~h} \mathrm{APF}$ pupae $(p<0.001$ ) (Fig. 2 B3,C3,F). At 9 h APF, Dlg staining had almost completely disappeared with only residual signals (Fig. 2D1). Meanwhile, staining signals of anti-Syt and anti-HRP showed greatly enlarged bouton size (from $6.91 \pm 0.33 \mu \mathrm{m}^{2}$ in third-instar larvae to $30.86 \pm 2 \mu \mathrm{m}^{2}$ in a $9 \mathrm{~h} \mathrm{APF}$ pupae; $p<$ 0.001 ) and decreased bouton number (from $30.73 \pm 0.95$ in third-instar larvae to $2.73 \pm 0.25$ in a 9 h APF pupae; $p<0.001$ ) (Fig. 2 D2,D3). To further analyze the degree of NMJ dismantling, we quantified the NMJ area positive for anti-Syt staining of larvae and pupae at different developmental stages. Compared with the 


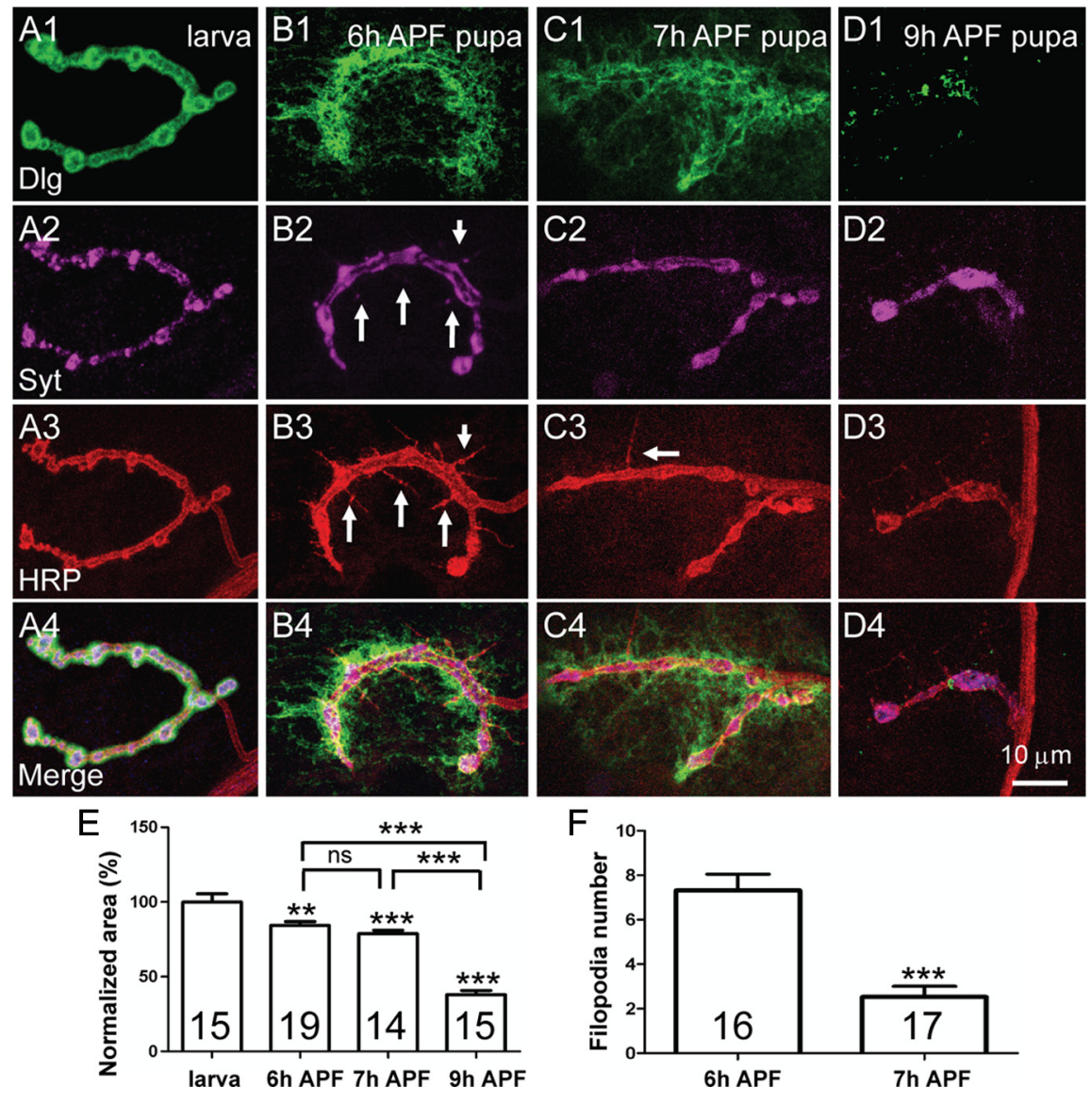

Figure 2. Distinct dismantling processes of presynaptic and postsynaptic elimination. Postsynaptic components become diffuse and eventually degraded, whereas SVs aggregate and presynaptic membrane retracts. $\boldsymbol{A}-\boldsymbol{D}$, Representative images of NMJ 4 from abdominal segment A3 of a third-instar larva (A), 6 h APF pupa $(\boldsymbol{B}), 7 \mathrm{~h} \mathrm{APF} \mathrm{pupa}(\boldsymbol{C})$, and $9 \mathrm{~h} \mathrm{APFpupa} \mathrm{(D).} \mathrm{NMJ} \mathrm{synapses} \mathrm{were}$ triply labeled by anti-Dlg (green), anti-Syt (magenta), and Texas Red-labeled anti-HRP (red). Anti-Dlg detects the postsynaptic scaffold protein DIg; anti-Syt labels SVs and anti-HRP labels the presynaptic membrane. A1-A4, A typical NMJ 4 terminal with beads-on-a-string boutons in a third-instar larva. B1-B4, Postsynaptic Dlg staining is diffused, whereas filopodia-like structures indicated by arrows in $\mathbf{B} 3$ are formed at $6 \mathrm{~h}$ APF. Anti-Syt staining shows larger aggregates compared with the discrete punctate staining seen at the larval NMJ (compare $\mathbf{B 2}, \mathbf{A 2}$ ). The arrows in $\mathbf{B 2}$ and $\boldsymbol{B} \mathbf{3}$ indicate Syt-positive signals overlapping with filopodia-like structures. C1-C4, Postsynaptic Dlg is more diffused at $7 \mathrm{~h} \mathrm{APF}$ and the number of filopodia-like structures has dramatically decreased compared with that at $6 \mathrm{~h}$ APF. D1-D4, Anti-DLG staining has almost disappeared by $9 \mathrm{~h}$ APF, whereas synaptic vesicles labeled by anti-Syt staining and presynaptic membrane labeled by anti-HRP staining are further aggregated and retracted to form a few large boutons. Scale bar, $10 \mu \mathrm{m}$. $\boldsymbol{E}, \boldsymbol{F}$, Statistical results of synaptic area positive for anti-Syt staining from third-instar larvae to $9 \mathrm{~h} \mathrm{APF}$ pupae $(\boldsymbol{E})$ and the number of filopodial structures detected by anti-HRP staining during synapse elimination $(\boldsymbol{F})$. The number of animals analyzed is indicated. "ns" denotes no significant difference; ${ }^{* *} p<0.05 ;{ }^{* * *} p<0.001$. Error bars indicate SEM.

NMJ area of third-instar larvae (normalized to 100), the NMJ areas of $6 \mathrm{~h}$ pupa $(84.35 \pm 2.47), 7 \mathrm{~h}$ pupa $(78.66 \pm 2.36)$, and $9 \mathrm{~h}$ APF pupa $(37.95 \pm 2.88)$ are gradually and significantly decreased $(p<0.01$ or 0.001$)$ (Fig. $2 E$ ).

In summary, postsynaptic Dlg was already diffused at $6 \mathrm{~h} \mathrm{APF,}$ reduced gradually, and was eventually eliminated at $9 \mathrm{~h}$ APF. Conspicuous presynaptic filopodial structures were observed at $6 \mathrm{~h} \mathrm{APF,}$ followed by SV aggregation and presynaptic plasma membrane retraction to form larger and fewer boutons at $9 \mathrm{~h} \mathrm{APF}$.

Postsynaptic elimination is characterized by diffuse Dlg staining, expanded localization of glutamate receptors, and progressive disassembly of the subsynaptic reticulum Previous studies have shown that the PDZ (postsynaptic density95/Discs large/zona occludens-1) domains of the postsynaptic scaffold protein Dlg control the localization of CD8-GFP-Shaker through their interaction with the $\mathrm{C}$ terminus of the potassium channel Shaker (Zito et al., 1999). To determine whether CD8-GFP-Shaker changes concomitantly with Dlg during synapse elimination, we followed both Dlg and CD8-GFP-Shaker by double labeling (Fig. $3 A$ ). This revealed that CD8-GFP-Shaker and anti-Dlg staining were precisely colocalized at larval NMJ (data not shown), and synchronously became diffuse at pupal NMJ synapses (Fig. 3A). Thus, CD8-GFPShaker provides an additional postsynaptic marker similar to anti-Dlg staining.

The Drosophila NMJ synapses contain two subtypes of postsynaptic ionotropic glutamate receptors A-type and B-type. The A-type and B-type receptors are composed of three shared subunits GluR IIC (also known as GluR III), GluR IID, and GluR IIE, and the subtype-specific GluR IIA and GluR IIB, respectively (Marrus et al., 2004; Featherstone et al., 2005; Qin et al., 2005). We then analyzed changes in the expression of different GluRs and the postsynaptic markers CD8-GFP-Shaker during synapse elimination. In thirdinstar larvae, anti-GluR IIA staining mostly overlapped with CD8-GFP-Shaker signals (Fig. $3 B$ ). However, at $7 \mathrm{~h} \mathrm{APF,}$ the distribution of postsynaptic CD8GFP-Shaker and GluR IIA was dramatically different (Fig. 3, compare C2, C1); CD8-GFP-Shaker signals were diffuse, but GluR IIA staining remained intense although its localization was more loose and the distribution area had expanded by $\sim 50 \%$ from 129 to $193 \mu \mathrm{m}^{2}(N \geq 7$; $p<0.05)$, compared with that of the larval NMJ synapses (Fig. 3, compare C2, B2). At $9 \mathrm{~h} \mathrm{APF}$, the anti-GluR IIA signals were greatly reduced (supplemental Fig. $1 B 1, E 1$, available at www. jneurosci.org as supplemental material). Double labeling showed that the subtypespecific GluR IIA and IIB as well as the shared subunit GluR IIC and the opposing active zone component Bruchpilot (recognized by monoclonal antibody NC82) are eliminated concomitantly (supplemental Fig. 1, available at www.jneurosci. org as supplemental material), although the two subtypes are differentially regulated during synapse development (Chen and Featherstone, 2005). These results demonstrate that the postsynaptic scaffold protein Dlg and GluRs are eliminated by different mechanisms.

To investigate the ultrastructural changes during synapse elimination, we next examined synaptic boutons of third-instar larvae and early pupae by EM. Presynaptic structures essential for neurotransmitter release at NMJ synapses include active zones with T bars and SVs (Fig. 3D) (Zhai and Bellen, 2004). The most prominent postsynaptic structure is the SSR, which consists of multiple layers of convoluted muscle plasma membrane (Fig. $3 D$ ). Compared with the third-instar larva, $6 \mathrm{~h}$ APF pupae had similar presynaptic structures. Normal T bar with docked SVs in a synapse from a $6 \mathrm{~h}$ APF pupa was observed (Fig. 3E). 
However, the postsynaptic SSR was more loose and vacuolized at $6 \mathrm{~h}$ APF compared with the tightly arranged SSR of the third-instar larva (Fig. 3, compare $E, D)$. Disassembly and vacuolization of the SSR are consistent with the diffuse localization of Dlg and CD8-GFPShaker observed by light microscopy (Figs. 2, 3).

Time-lapse imaging reveals retrograde transport of synaptic vesicles and retraction of presynaptic membrane To visualize the dynamic process of synapse dismantling in detail, we performed live-imaging microscopy. Drosophila NMJs were labeled with Syt-eGFP, a fluorescent marker suitable for studying SV dynamics in vivo (Zhang et al., 2002). Pupae at specified developmental stages were dissected and maintained in modified HL3 solution; the dissected animals remained alive at least for $2 \mathrm{~h}$ under the culture conditions. The dynamics of Syt-eGFP-labeled SVs in a synaptic terminal during synapse elimination is shown in Figure $4 A$. At $8 \mathrm{~h} 20 \mathrm{~min}$ APF, there was a large bleb (up to $8 \mu \mathrm{m}$ in diameter) of eGFP-labeled SVs at the distal tip of the lower synaptic branch (Fig. 4A1) and no SVs were observed in the innervating axons. Fifteen minutes later, eGFP-labeled SVs were observed to be retrogradely transported back into the innervating axon (indicated by a green triangle in Fig. 4A2). At the same time, SVs located at the distal ends of the NMJ terminals moved toward the nerve-muscle contact site (arrowed in Fig. 4A2). At 9 h 40 min APF, eGFP-labeled SVs

in the upper branch of the NMJ terminal almost completely disappeared (indicated by a yellow triangle in Fig. 4A6); meanwhile, more SVs moved into the innervating axons and further away from the nerve-muscle contact site, indicating retrograde axonal transport of SVs during synapse elimination.

In addition to the dramatic SV changes, we also monitored the dynamics of presynaptic membrane labeled by SNAP25-YFP under the control of the motor neuron-specific OK6-Gal4 during synapse elimination (Fig. 4C). SNAP25 is a presynaptic plasma membraneassociated protein and is a core component of the SNARE (soluble $N$-ethylmaleimide-sensitive factor attachment protein receptor) complex that mediates membrane fusion (Vilinsky et al., 2002). One prominent feature we observed was the filopodia-like structures emanating from the presynaptic membrane (Fig. 4C1-C5), similar to that detected by anti-HRP staining (Fig. 2B3,C3). The presynaptic membrane then retracted from the distal ends toward the nervemuscle contact site (Fig. 4C, compare C5, C1) and may be eventually detached from the postsynaptic domains.

Blockade of axonal transport inhibits presynaptic elimination Time-lapse imaging shown above revealed retrograde axonal transport of SVs during presynaptic elimination (Fig. 4). To further investigate a role for retrograde axonal transport in the process, we examined synapse dismantling in Dhc64C mutants and in animals expressing a dominant-negative mutant of glued in pre- synaptic motor neurons. Dhc64C encodes a cytoplasmic dynein heavy chain and mutations in Dhc64C disrupt fast retrograde transport (Pilling et al., 2006). glued encodes a $150 \mathrm{kDa}$ protein that is part of the dynactin complex that activates dynein activity and links the motor to its cargo. The glued $^{1}$ mutation encodes a C-terminally truncated protein without cargo binding ability that acts as a dominant negative and disables retrograde transport of motor dynein (Allen et al., 1999). Both Dhc64C and Glued function in retrograde axonal transport (Allen et al., 1999; Pilling et al., 2006).

Presynaptic elimination was delayed in both Dhc64C mutants and animals expressing glued $^{1}$ in presynaptic neurons. We first confirmed that the NMJ synapses in a third-instar larva of Dhc64C $6-19 / D h c 64 C^{4-10}$ hypomorphic heterozygous mutants (Dhc64C nulls are embryonic lethal, thus precluding synapse elimination analysis during metamorphosis) and animals with presynaptic expression of glued $^{1}$ were mostly normal and indistinguishable from wild type (data not shown). Indeed, even at $7 \mathrm{~h}$ APF, NMJ synapses appeared normal in both Dhc64C mutants and animals expressing glued $^{1}$ (Fig. 5A1-C3). However, in contrast to the dramatic aggregation and withdrawal of SVs labeled by anti-Syt in wild-type pupae at $9 \mathrm{~h}$ APF (Fig. 5D2), we observed no obvious change of SVs in Dhc64C mutants (Fig. 5E2) and in animals expressing glued $^{l}$ (Fig. 5F2), although postsynaptic Dlg was diffuse and almost completely disappeared as in the wild type 

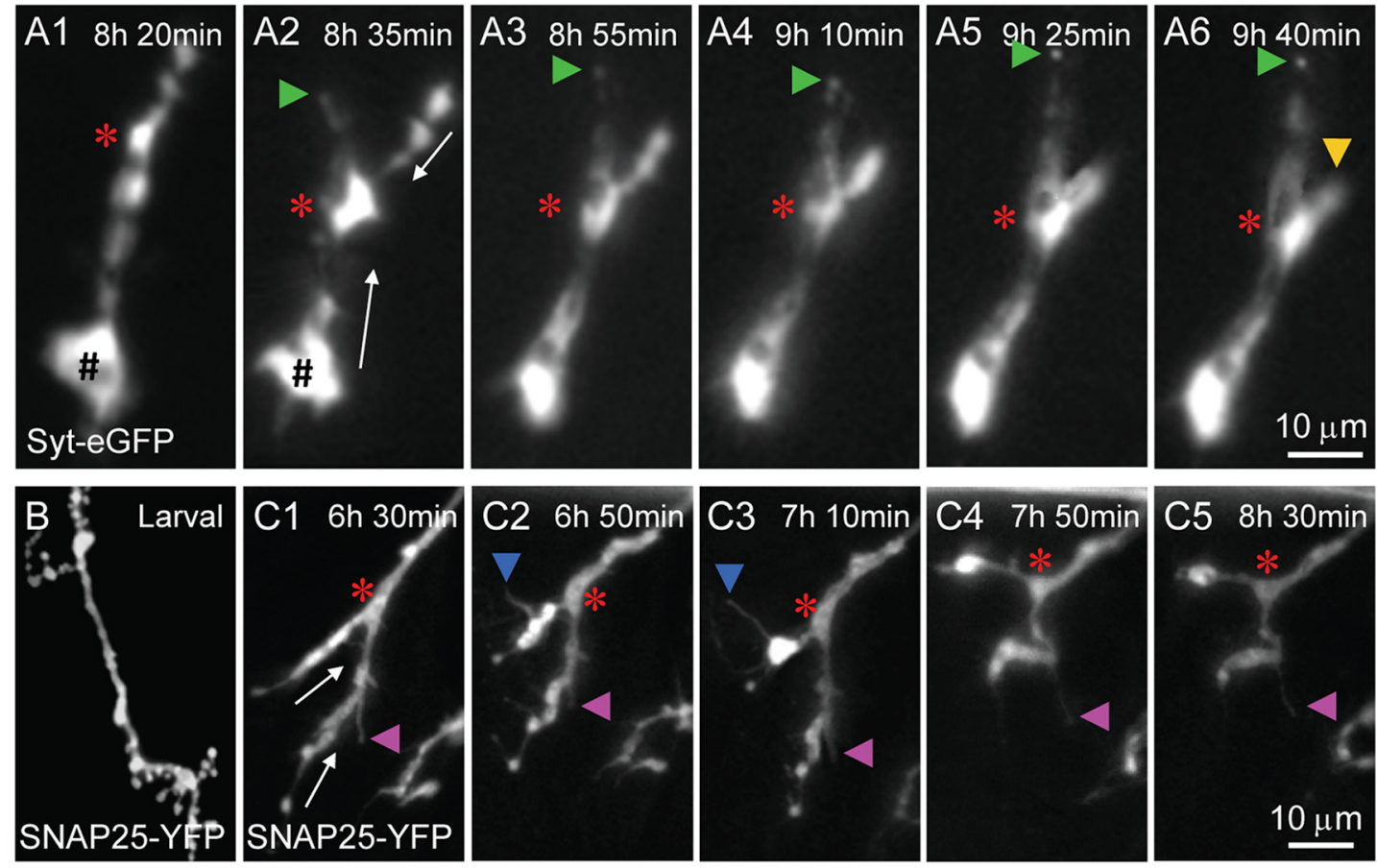

Figure 4. Time-lapse imaging of presynaptic changes during NMJ synapse elimination. A1-A6, Time-lapse imaging of SVs labeled by Syt-eGFP under the control of pan-neuronal elav-Gal4 shows aggregation, retraction, and retrograde axonal transport of SVs from $8 \mathrm{~h} 20 \mathrm{~min}(\boldsymbol{A} 1)$ to $9 \mathrm{~h} 40 \mathrm{~min}$ (A6) APF. The red asterisk indicates the site of nerve-muscle contact (proximal end of the synaptic terminals). The green triangles indicate SVs retrograde transporting in axons. The white arrows indicate the direction of retrograde SV transport in synaptic terminals toward the nerve-muscle contact site; the yellow triangle indicates the tip of a retracted synaptic branch. SV aggregates indicated by the hash sign (\#) are as large as $8 \mu \mathrm{m}$ in diameter $(\boldsymbol{A} \mathbf{1}, \boldsymbol{A 2})$. Scale bar, $10 \mu \mathrm{m}$. $\boldsymbol{B}, \boldsymbol{C}$, Presynaptic membranes labeled by SNAP25-YFP under the control of motor neuron specific OK6-Gal4 from a larva (B) and early pupa (C1-C5). Presynaptic terminals were retracted during the observation period from $6 \mathrm{~h} 30 \mathrm{~min}$ to $8 \mathrm{~h} 30 \mathrm{~min}$ APF (C1-C5). Dynamic filopodia-like structures are indicated by blue and pink triangles; the site of nerve-muscle contact is indicated by a red asterisk; the white arrows in $\mathbf{C}$ denote the direction of retraction of the presynaptic membrane. Scale bar, $10 \mu \mathrm{m}$.

(compare E1, F1, with D1). Statistically, compared with the normalized synaptic area positive for anti-Syt staining of wild-type pupae at $9 \mathrm{~h} \mathrm{APF}(37.95 \pm 2.88 \%$ of the larval synapse area), Dhc64C mutants and animals expressing glued $^{1}$ showed significantly larger synaptic areas $(87.85 \pm 2.96$ and $91.56 \pm 8.58 \%$ of the corresponding larval synapse areas for Dhc64C mutants and glued $^{1}$-expressing animals, respectively; $p<0.001$ ) (Fig. 5I). Immunostaining with NC82, a mouse antibody against Bruchpilot, also showed obviously delayed elimination of the active zone component in animals expressing glued $^{l}$ (Fig. 5, compare $H, G$ ). These data together demonstrate that retrograde axonal transport is required for presynaptic but not postsynaptic elimination.

\section{Synapse elimination is arrested by postsynaptic inhibition of} the ubiquitination pathway or apoptosis

The ubiquitination pathway plays an important role in synapse growth (DiAntonio and Hicke, 2004). Mutations in the ring domain ubiquitin E3 ligase Highwire result in exuberant NMJ synapse growth (Wan et al., 2000; Collins et al., 2006). Similarly, downregulation of the ubiquitination pathway by overexpression of the deubiquitinating protease Fat Facets or the yeast deubiquitinating protease UBP2 in presynaptic neurons by elav-Gal4 also produced a dramatic increase in synapse growth (DiAntonio et al., 2001). More importantly, the ubiquitination pathway has been shown to be involved in axonal and dendritic pruning (Watts et al., 2003; Kuo et al., 2005, 2006). To investigate a possible role of the ubiquitination pathway in synapse elimination, we first examined NMJ synapses in highwire mutants and found that synapse elimination proceeded normally as in wild type (supplemental Fig. 2, available at www.jneurosci.org as supplemental material).
Overexpression of the yeast deubiquitinating protease UBP2 in presynaptic neurons by OK6-Gal4 also resulted in no delay of synapse elimination as seen in highwire mutants (data not shown). However, when UBP2 was overexpressed in the postsynaptic muscles by BG57Gal4, synaptic growth was normal but synaptic elimination was delayed (Fig. 6, compare $B$ with $A$, and $F$ with $E$ ), as postsynaptic Dlg was still tightly localized (Fig. 6B1) instead of diffused in wild type at $7 \mathrm{~h} \mathrm{APF} \mathrm{(Fig.} \mathrm{6A1),} \mathrm{and} \mathrm{no} \mathrm{presynaptic} \mathrm{filopodia-like} \mathrm{structures} \mathrm{were}$ formed at $7 \mathrm{~h} \mathrm{APF} \mathrm{(Fig.} \mathrm{6B2).}$

Ubiquitination and apoptotic pathways are known to coordinately control neuronal pruning in Drosophila (Kuo et al., 2006); we therefore sought to investigate whether apoptosis is also involved in synaptic elimination. To test this possibility, we overexpressed caspase inhibitors P35 and Diap1 (Drosophila inhibitor of apoptosis protein 1, an E3 ligase that antagonizes caspase Dronc activity) in presynaptic neurons by OK6-Gal4 or in postsynaptic muscles by BG57-Gal4. Overexpression of P35 and Diap1 in presynaptic neurons had no obvious effect on synapse elimination (data not shown). However, synapse elimination was strongly arrested at 7 and $9 \mathrm{~h} \mathrm{APF}$ when the two apoptosis inhibitors P35 and Diap1 were overexpressed postsynaptically (Fig. 6, compare $C, D$, with $A, G$, and $H$ with $E$ ). Overexpression of Diap1 and $\mathrm{P} 35$ produced a similar degree of synapse elimination delay (Fig. 6, compare $C$ and $D, G$ and $H$ ) but apparently a stronger effect than that of UBP2 overexpression (Fig. 6, compare $G, H$, with $F$ ). Immunostaining of $9 \mathrm{~h}$ APF pupae with anti-GluR IIA and anti-GluR IIB also showed delayed elimination of GluRs when ubiquitination or apoptotic pathway was inhibited in muscles (supplemental Fig. 3, available at www.jneurosci.org as supplemental material). The severity of synapse elimination delay 
was dependent on the expression levels of P35 and Diap1 because higher expression of the two apoptosis inhibitors by the stronger driver BG57-Gal4 produced a more pronounced delay in synapse elimination (Fig. $6 C, D, G, H$ ) than did the weaker driver $M H C-G a l 4$ (data not shown).

In summary, inhibition of the ubiquitination pathway or apoptosis in presynaptic neurons had no apparent effect on synapse elimination, but blockade of the ubiquitination pathway or apoptosis in postsynaptic muscles arrested both presynaptic and postsynaptic elimination, suggesting a crucial role for the postsynaptic side in synapse elimination.

Presynaptic and postsynaptic blockade of ecdysone signaling differentially delays synapse elimination

The results presented above show that the postsynaptic side plays a crucial role in synapse elimination. Ecdysone signaling is the master regulator of neuronal remodeling (Lee et al., 2000; Zheng et al., 2003; Kuo et al., 2005; Williams and Truman, 2005). To better understand the differential roles of the presynaptic and postsynaptic sides in synapse elimination, we blocked ecdysone signaling in either presynaptic or postsynaptic cells by expression of dominant-negative ecdysone receptor isoforms EcR.A and EcR.B1 using the UAS-Gal4 system (Brand and Perrimon, 1993; Cherbas et al., 2003; Awasaki et al., 2006). The dominant-negative mutant has only $1 \%$ of the activity of the wildtype receptor in activating target gene transcription (Cherbas et al., 2003). Higher expression of $E c R \cdot B 1^{D N}$ in muscles by the stronger driver BG57-Gal4 resulted in first-instar larval lethality, but lower expression of $E c R \cdot B 1^{D N}$ by the moderate driver $M H C$-Gal4 led to pupal lethality, allowing us to examine its effect on synapse elimination in early metamorphosis.

Compared with the diffuse localization of Dlg in the $6 \mathrm{~h}$ wild-type pupa (Fig. $7 A$ ), tightly arranged Dlg was present in a $6 \mathrm{~h}$ APF pupa expressing EcR.B1 $1^{D N}$ in muscles by $M H C$-Gal4 (Fig. 7B1), whereas the pattern of presynaptic anti-Syt staining was comparable with that of wild type (Fig. 7, compare B2, A2). Overexpression of another independent dominant-negative receptor $E c R . A^{D N}$ produced a similar but weaker delay of synapse elimination than that by the overexpression of $E c R \cdot B 1^{D N}$ (data not shown), confirming that the effect is specifically caused by postsynaptic disruption of the ecdysone signaling. We next expressed $E c R . A^{D N}$ in presynaptic neurons by OK6-Gal4 to block ecdysone signaling and found no delay in synapse elimination (Fig. 7C1) (expression of $E c R \cdot B 1^{D N}$ by OK6-Gal4 led to larval lethality precluding synapse elimination analysis during metamorphosis). Thus, inhibition of ecdysone signaling in presynaptic neurons did not obviously affect synapse dismantling, but inhibition
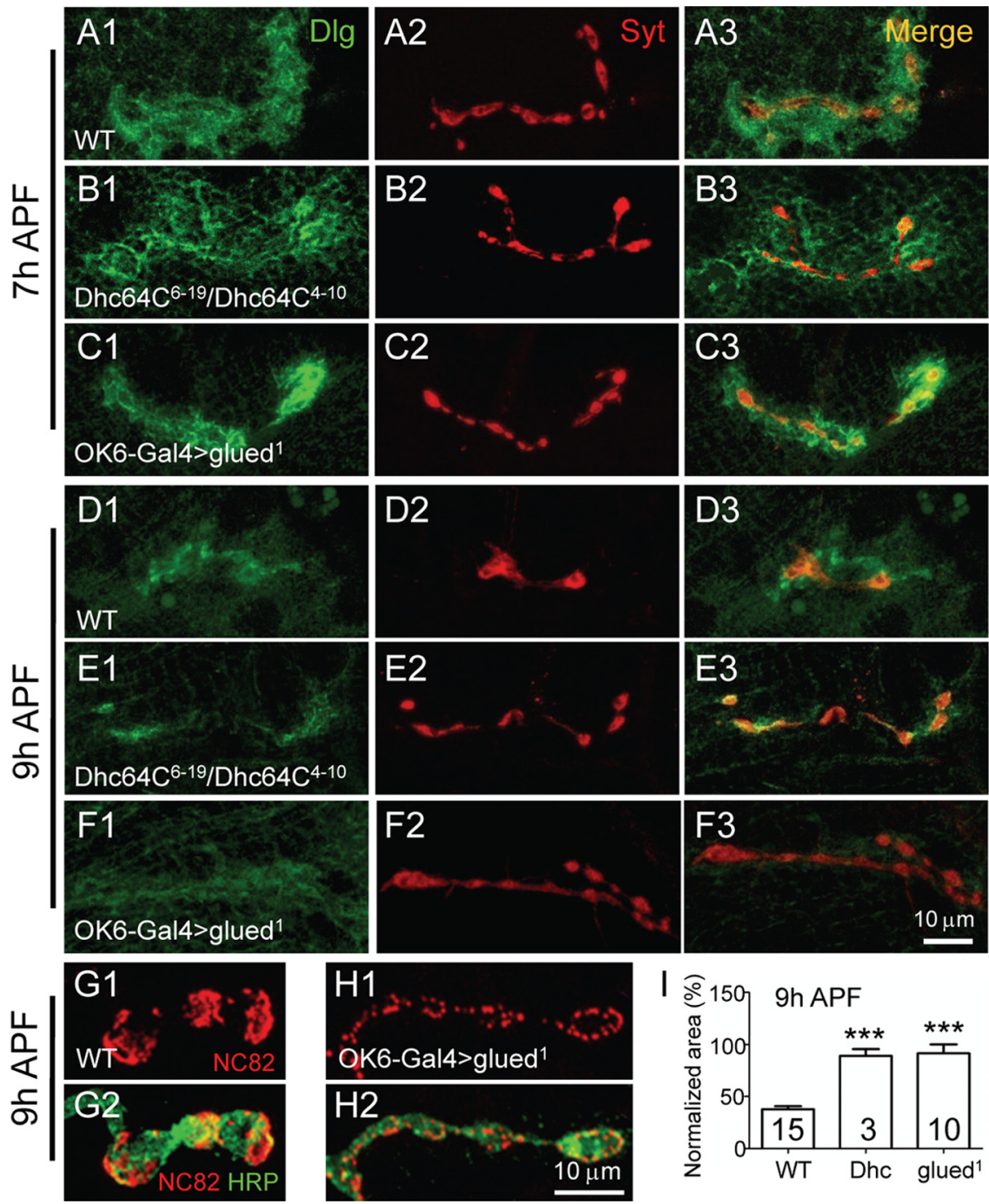

Figure 5. Retrograde axonal transport is crucial for presynaptic elimination. NMJ synapses of different genotypes were doubly labeled with anti-Dlg (green) and anti-Syt (red). At $7 \mathrm{~h}$ APF, NMJ synapses in Dhc64C ${ }^{6-19} / D h c 64 C^{4-10}$ heteroallelic mutants (B1OK6-Gal4 (C1-C3) resembled the wild type (A1-A3). However, at $9 \mathrm{~h} \mathrm{APF,} \mathrm{presynaptic} \mathrm{elimination} \mathrm{was} \mathrm{clearly} \mathrm{delayed} \mathrm{in} \mathrm{the}$

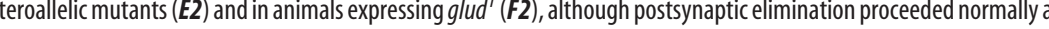
of the active zone component Bruchpilot detected by NC82 staining $(\boldsymbol{H})$ compared with wild type $(\boldsymbol{G})$. $\boldsymbol{I}$, Quantification of bouton area positive for anti-Syt staining in $9 \mathrm{~h}$ APF pupae of $D h$ c mutants and animals expressing glud ${ }^{7}$. The number of animals analyzed is indicated. ${ }^{* *} p<0.001$. Error bars indicate SEM.

of the ecdysone signaling in postsynaptic muscles delayed postsynaptic elimination at $6 \mathrm{~h}$ APF.

Because synapse elimination, particularly the presynaptic elimination, is more advanced and easier to score at $9 \mathrm{~h}$ AFP than at $6 \mathrm{~h} \mathrm{APF}$, we further examined NMJ synapses in $9 \mathrm{~h} \mathrm{APF}$ pupae when ecdysone signaling was blocked presynaptically and postsynaptically. In a $9 \mathrm{~h} A P F$ pupa expressing $E c R \cdot B 1^{D N}$ postsynaptically by $\mathrm{MHC}$-Gal4, both presynaptic and postsynaptic elimination was completely blocked (Fig. 7, compare E, D) with synaptic localization of GluR IIA and IIB intact (Fig. 7G). In addition, the integrity of muscles was protected by the genetic manipulation as evidenced by phalloidin staining (Fig. 7, compare $K, I)$. However, in $9 \mathrm{~h}$ APF pupae expressing EcR. $A^{D N}$ in presynaptic neurons, there were no signs of presynaptic dismantling such as SV aggregation, decreased bouton number, or enlarged bouton size (Fig. 7F2), but the postsynaptic dismantling 


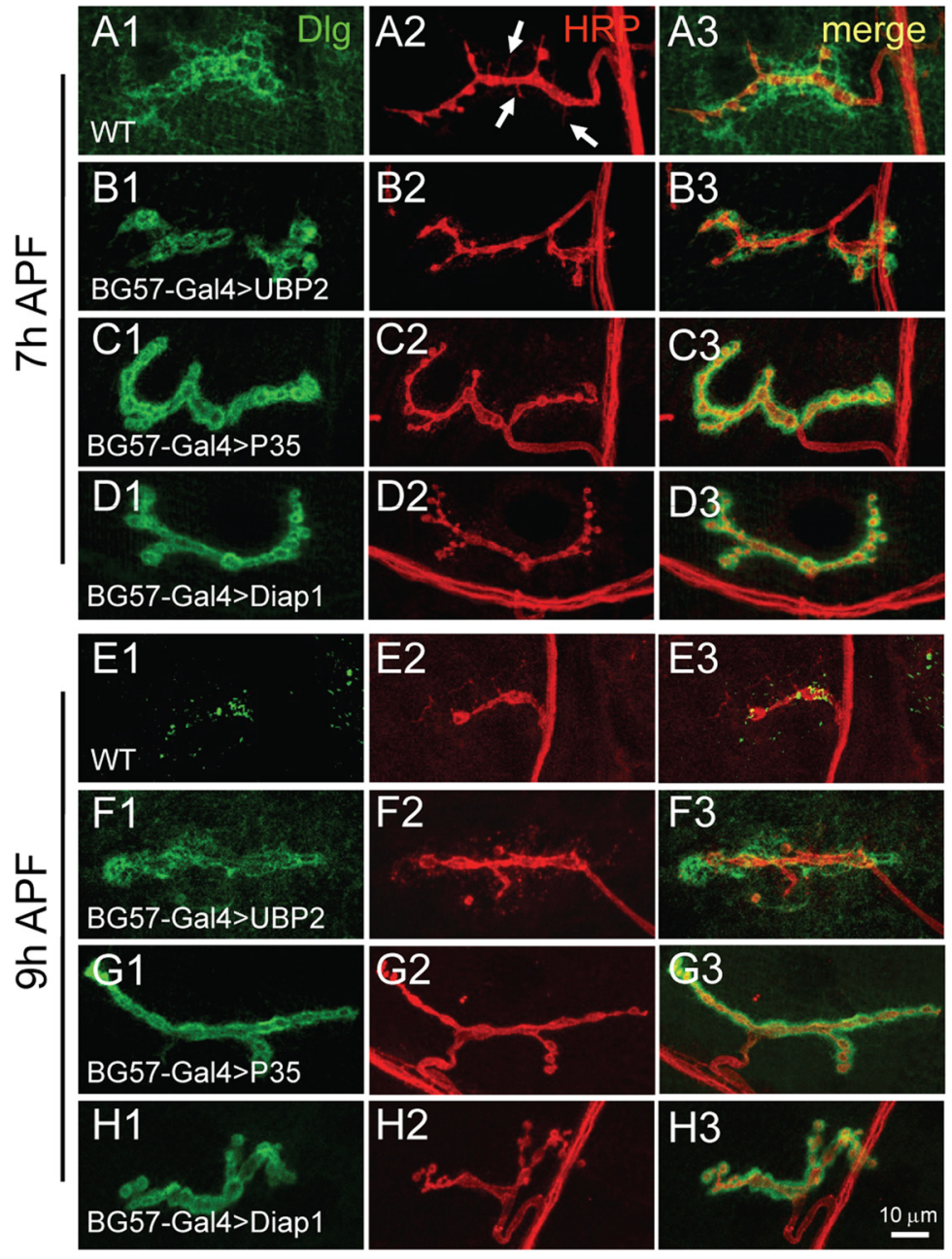

Figure 6. Blockade of ubiquitination pathway or apoptosis in postsynaptic muscle cells arrests synaptic elimination. NMJ synapses doubly labeled with anti-Dlg (green) and anti-HRP (red) in wild-type pupae $(\boldsymbol{A}, \boldsymbol{E})$ and in pupae with postsynaptic expression of the yeast deubiquitinating protease UBP2 $(\boldsymbol{B}, \boldsymbol{F})$, the apoptosis inhibitor $\mathrm{P35}(\boldsymbol{C}, \boldsymbol{G})$, and the apoptosis inhibitor Diap1 $(\boldsymbol{D}, \boldsymbol{H})$ driven by $B G 57-G a 14$. NMJ synapses from 7 and $9 \mathrm{~h}$ APF pupae are presented in $\boldsymbol{A}-\boldsymbol{D}$ and $\boldsymbol{E}-\boldsymbol{H}$, respectively. Filopodial structures emanating from a wild-type NMJ terminal at $7 \mathrm{~h}$ APF are indicated by arrows (A2). Scale bar, $10 \mu \mathrm{m}$.

occurred normally (Fig. 7F1). Statistically, the synaptic area positive for anti-Syt staining in wild-type pupae at $9 \mathrm{~h} \mathrm{APF} \mathrm{is} 37.95 \%$ of that in third-instar larvae (Fig. $7 H$ ). However, the synaptic area in $9 \mathrm{~h} \mathrm{APF}$ pupae expressing $E c R . A^{D N}$ is $70.06 \%$ of that in the corresponding larvae, significantly higher than that in wild type $(p<0.001)$ (Fig. 7H). In summary, inhibition of ecdysone signaling in presynaptic neurons arrested presynaptic elimination only, but inhibition of the ecdysone pathway in postsynaptic muscles arrested both presynaptic and postsynaptic elimination, indicating that the postsynaptic side plays an instructive role in synapse elimination.

\section{Glial cells are not involved in NMJ synapse elimination}

Previous studies have shown that glial infiltration and engulfment participate in axonal elimination in the Drosophila brain under physiological and pathological conditions (Awasaki and Ito, 2004; Watts et al., 2004; MacDonald et al., 2006). To address whether glia are also involved in peripheral NMJ synapse elimination during metamorphosis, we labeled glia with a GFP marker driven by the glia specific Repo-Gal4. Peripheral glia extend along axons to the nerve-muscle contact site (Fig. $8 A$ ), consistent with a previous report (Sepp et al., 2000). If glia were involved in synapse elimination, colocalization or close proximity of synaptic components and GFPlabeled glial cells would be expected. However, SVs were not engulfed by GFPlabeled glia at $9 \mathrm{~h} \mathrm{APF}$, because there was no overlapping labeling of Syt-positive SVs and glial cells (Fig. 8B). To further examine whether glia are involved in synapse elimination, we blocked ecdysone signaling specifically in glia by expressing $E c R \cdot A^{D N}$ or EcR.B1 $1^{D N}$ by Repo-Gal4. The animals expressing $E c R^{D N}$ in glia were pharate lethal, indicating the efficacy of the tissue-specific blockade of ecdysone signaling, but showed normal NMJ synapses elimination as wild type in a 9 h APF pupa (Fig. 8, compare C2, C1). In addition, glia-specific expression of $s h i^{\text {ts }}$ at restrictive temperature blocking endocytosis also resulted in no delay in synapse elimination (data not shown). These results together indicate that glial cells are not involved in NMJ synapse elimination.

\section{Discussion}

Distinct presynaptic and postsynaptic dismantling mechanisms

The Drosophila NMJ is an attractive model system for studying synaptogenesis (Collins and DiAntonio, 2007) but has only rarely been exploited to study synapse elimination (Eaton et al., 2002; Goda and Davis, 2003). In the present study, we used the Drosophila NMJ to study synapse elimination in the early stages of metamorphosis during which extensive synapse elimination occurs. Using confocal microscopy, live imaging, and electron microscopy, we have unveiled distinct presynaptic and postsynaptic dismantling processes (Table 1). Presynaptic elimination is characterized by the formation of prominent filopodial structures (Figs. 2, 4 ; Table 1). The presynaptic membrane then retracts toward the nerve-muscle contact site with decreased bouton number and enlarged bouton size, accompanied by SV aggregation and retrograde axonal transport of SVs. It is worth pointing out that the precise timing of synapse dismantling revealed by immunostaining (Fig. 2) and live imaging (Fig. 4) is different, and this is probably attributable to the fact that the samples were analyzed under different conditions. For example, animals were kept at $25^{\circ} \mathrm{C}$ for immunostaining but were maintained at $20^{\circ} \mathrm{C}$ during live imaging. It is well known that filopodia are present in growth cones and play an important role in neurite outgrowth. The filopodialike structures we observed during synapse elimination (Figs. 2, 4) presumably sense and explore the environment. Our data also demonstrate for the first time that retrograde axonal transport plays an important role in presynaptic elimination (Figs. 4,5 ). It is expected that the retrogradely transported synaptic constituents are reused to form adult-specific synaptic connections, although, to our knowledge, the final fate of the motor neuron MN4a innervating muscle 4 has not been determined. During the metamorphic period from 4 to $11 \mathrm{~h} \mathrm{APF}$ we examined for the complete synapse elimination (Table 1), we have observed nei- 

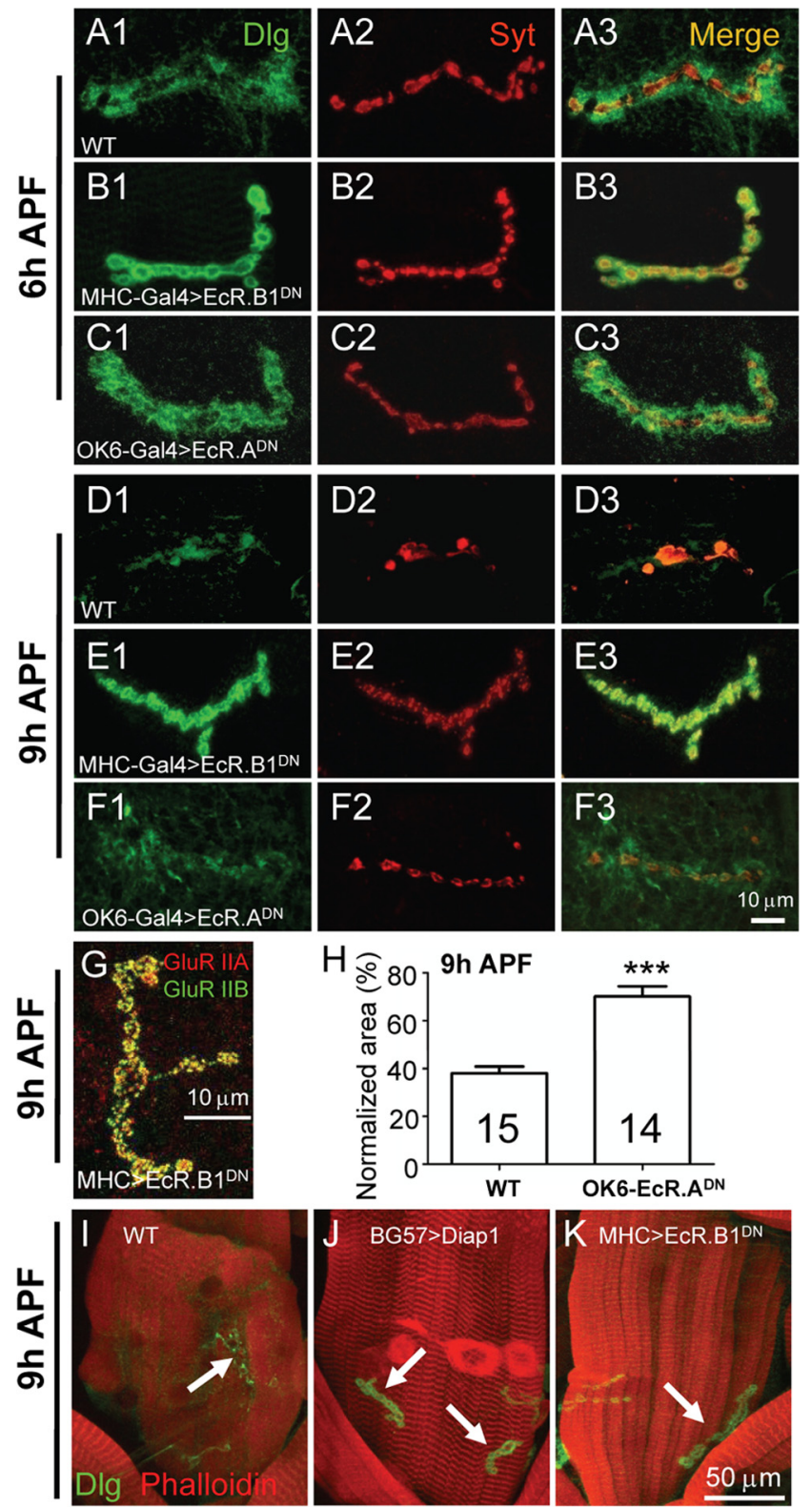

Figure 7. Inhibition of ecdysone signaling by presynaptic or postsynaptic expression of a dominant-negative ecdysone receptor differentially delays synapse elimination. NMJ synapses from different genotypes were double-stained with anti-Dlg (green) and anti-

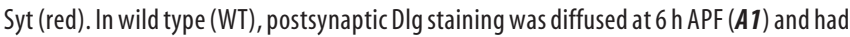
almost disappeared at $9 \mathrm{~h}$ APF (D1). The pattern of anti-Syt staining of WT at $6 \mathrm{~h}$ APF (A2) appeared similar to that of the larval NMJ, but anti-Syt staining signal at $9 \mathrm{~h}$ APF was markedly decreased (D2). Blockade of ecdysone signaling in the postsynaptic muscles by expression of a dominant-negative ecdysone receptor $E C R . B 1^{D N}$ driven by $M H C$-Gal 4 led to an arrest of postsynaptic elimination at $6 \mathrm{~h}$ APF (B1-B3), and a complete arrest of both presynaptic and postsynaptic elimination at $9 \mathrm{~h}$ APF (E1-E3). However, inhibition of ecdysone signaling in presynaptic motor neurons by expressing the dominant-negative $E c R . A^{D N}$ driven by $0 K 6-G a / 4$ showed no obvious delay in synapse elimination at $6 \mathrm{~h} \operatorname{APF}(\boldsymbol{C})$, but a complete arrest of presynaptic ( $F 2$ ) but not postsynaptic (F1) elimination at $9 \mathrm{~h}$ APF. Scale bar, $10 \mu \mathrm{m}$. G, Unaltered expression of GluR IIA and GluR IIB at synapses in a $9 \mathrm{~h}$ APF pupa expressing $E C R . B 1^{D N}$ in muscles. $\boldsymbol{H}$, Quantification of bouton area positive for antiSyt staining in $9 \mathrm{~h}$ APF pupae expressing $E C R . A^{D N}$ in presynaptic neurons. The number of animals analyzed is indicated. ${ }^{* * *} p<0.001$. Error bars indicate SEM. $\boldsymbol{I}-\boldsymbol{K}$, Inhibiting apoptosis or ecdysone signaling in postsynaptic muscles delays destruction of muscles stained by phalloidin (red; compare $\boldsymbol{J}$ and $\boldsymbol{K}$ with $\boldsymbol{I}$ ) and the disassembly of Dlg (green). The arrows indicate NMJ 4 synapses in $\boldsymbol{I} \boldsymbol{K}$.

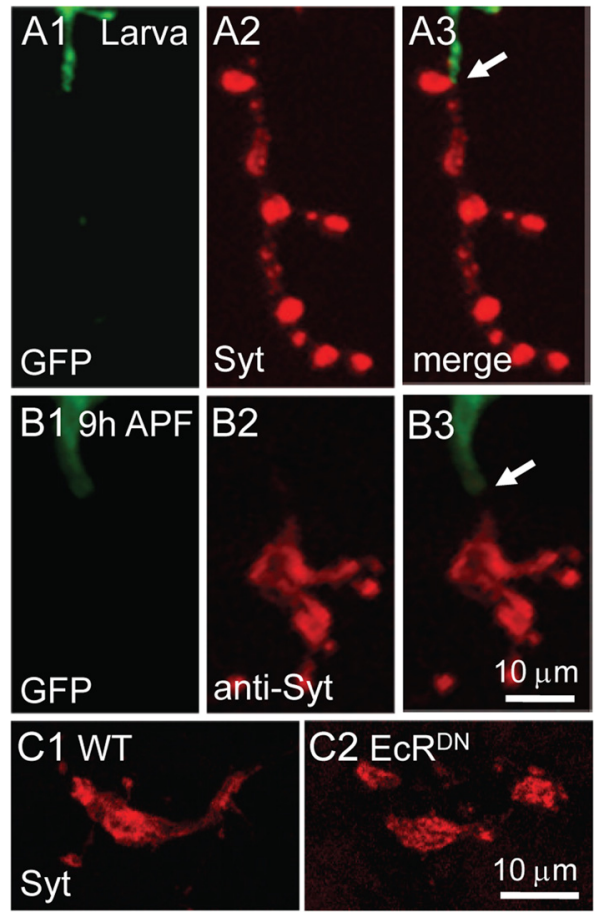

Figure 8. Glial cells are not involved in NMJ synapse dismantling. $A, B$, Glial cells are labeled with GFP (green) driven by the glial-specific Repo-Gal4, whereas presynaptic vesicles are labeled with anti-Syt (red). A1-A3 represent fully developed larval NMJ synapses, whereas B1-B3 show NMJ synapses in a $9 \mathrm{~h}$ APF pupa. No apparent overlap of glial cells and Syt-positive SVs was observed at $9 \mathrm{~h} A P F(B 1-B 3)$. The nerve-muscle contact site is indicated by an arrow in $A 3$ and $B 3$. C1 C C2, Anti-Syt staining of NMJ synapses in a $9 \mathrm{~h}$ wild-type APF pupa (C1) and a pupa with $E C R . A^{D N}$ overexpression by Repo-Gal4 (C2). No synapse dismantling delay was observed when ecdysone signaling was inhibited in glial cells (compare (2, C1). Scale bar, $10 \mu \mathrm{m}$.

ther disrupted synaptic microtubules as reported for the local synapse disassembly in Drosophila larvae (Eaton et al., 2002) nor an axonal "retraction bulb" as seen during mammalian NMJ synapse elimination; a retraction bulb appears when a presynaptic terminal is detached mostly or completely from the postsynaptic specialization (Gan and Lichtman, 1998; Bishop et al., 2004; McCann et al., 2007).

The first signs of postsynaptic elimination were the blurred and diffuse localization of postsynaptic markers Dlg and CD8GFP-Shaker at $4 \mathrm{~h} \mathrm{APF}$, followed by a more expanded distribution of GluRs and vacuolization of SSR in $6 \mathrm{~h}$ APF pupae (Figs. 2, 3; supplemental Fig. 1, available at www.jneurosci.org as supplemental material). The postsynaptic components of Dlg and CD8GFP-Shaker were almost completely eliminated at $9 \mathrm{~h}$ APF (Table 1). Completion of the synapse dismantling process starting from the diffusion of postsynaptic Dlg at $4 \mathrm{~h} \mathrm{APF}$ takes $\sim 7 \mathrm{~h}$ (Table 1 ). It is remarkable to note that the patterns of elimination of postsynaptic Dlg and GluRs are different; Dlg is eliminated by a diffusion-degradation process, whereas no diffusion of GluRs was observed before degradation (Fig. 3; supplemental Fig. 1, available at www.jneurosci.org as supplemental material), indicating that they are eliminated by different mechanisms. The differential elimination of Dlg and GluRs is consistent with the previous finding that the synaptic localization of GluR IIA is independent of Dlg (Chen and Featherstone, 2005). Interestingly, as for Drosophila GluRs, mammalian NMJ postsynaptic acetylcholine receptors are eliminated without the intermediate process of diffusion (Gan and Lichtman, 1998; Bishop et al., 2004; McCann et al., 2007). 
Synapse elimination is distinct from axonal and dendritic pruning. In Drosophila, the pruning of axonal and dendritic processes during metamorphosis closely resembles the pathological process of Wallerian degeneration, a process in which part of the axon separated from the nucleus of the neuron degenerates. In both axonal pruning of central mushroom body neurons and dendritic elimination of peripheral sensory neurons, severing of neuronal processes is preceded by microtubule depolymerization and followed by cytoplasmic blebbing and degeneration (Watts et al., 2003, 2004; Williams and Truman, 2005; Awasaki et al., 2006; Kuo et al., 2006). It is conceivable that synapse elimination and pruning of neuronal processes are closely interconnected, but the time course of the two discrete processes has yet to be determined. It has been shown that specific E2/E3 ubiquinating enzymes and caspases (i.e., UbcD1-Diap1-Dronc) are involved in dendritic pruning (Kuo et al., 2006). But it is unknown whether those molecules also participate in NMJ synapse elimination. However, we have demonstrated that disruption of ubiquitination and apoptosis pathways on the postsynaptic side arrests synaptic elimination (Fig. 6). It will be of great interest to identify the specific ubiquinating enzymes and caspases that participate in synapse dismantling during metamorphosis. It is worth noting that glial cells play an important role in axonal pruning of mushroom body neurons and olfactory receptor neurons (Awasaki and Ito, 2004; Watts et al., 2004; Awasaki et al., 2006; MacDonald et al., 2006). However, we found no evidence to suggest that glial cells play a role in NMJ synapse elimination (Fig. 8).

\section{Local synapse disassembly versus elimination of whole synaptic terminals}

Davis and colleagues (Eaton et al., 2002) first reported synapse disassembly or instability in Drosophila NMJ terminals. However, the process they studied is fundamentally different from that studied here in several aspects. First, they studied local synapse disassembly, the disassembly of distal synaptic boutons or a branch of the whole synaptic terminals of a motor neuron, a process that occurs during synapse growth in larval development, whereas we studied the elimination of complete NMJ 4 synapses in synchrony with muscle histolysis during metamorphosis. Also, the synapse elimination we report here is different from that of mammalian NMJ synapses; the former involves muscle destruction, whereas the latter does not. Second, the processes of local versus general synapse disassembly are different. In local synapse disassembly, presynaptic dismantling precedes postsynaptic dismantling: the presynaptic microtubule cytoskeleton retracts first, followed by the elimination of synaptic release machinery (i.e., the vesicle-associated protein synapsin) and ultimately the disassembly of the postsynaptic apparatus including the postsynaptic GluRs and the scaffold Dlg (Eaton et al., 2002). However, in the elimination of complete synaptic terminals during metamorphosis, postsynaptic dismantling starts first, followed by presynaptic dismantling (Table 1). Third, disrupting the dynactin complex destabilizes local synapses, leading to more synaptic "footprints" that are defined as the withdrawal of presynaptic components from clearly defined postsynaptic specialization containing Dlg (Eaton et al., 2002). They argued that the dynactin complex functions locally within presynaptic terminals to maintain synapse stability. Although we have not examined synaptic footprints during metamorphosis, we report here that disrupting the dynactin complex in presynaptic neurons delays presynaptic dismantling specifically, whereas the postsynaptic components disassemble normally, indicating that dynactin-mediated retrograde axonal transport is required for presynaptic elimination (Fig. 5). These results demonstrate that the dynactin complex functions differently in distinct cellular contexts.

\section{Postsynaptic signals instruct presynaptic elimination}

Two independent lines of evidence indicate that the postsynaptic rather than presynaptic side plays an instructive role in synapse elimination during metamorphosis. First, immunostaining showed that postsynaptic dismantling precedes presynaptic elimination by $\sim 1 \mathrm{~h}$ (Table 1 ). Second, blockade of retrograde axonal transport and ecdysone signaling specifically in presynaptic neurons delayed presynaptic dismantling only but postsynaptic dismantling proceeded normally. However, inactivation of ecdysone signaling, ubiquitination, or apoptosis pathways in postsynaptic muscles arrested both presynaptic and postsynaptic dismantling (Figs. 5-7; supplemental Fig. 3, available at www. jneurosci.org as supplemental material). We note that synapse elimination is closely correlated with muscle destruction (Fig. 7). Indeed, muscle histolysis might be the primary cause of NMJ synapse elimination during metamorphosis. These results together indicate that postsynaptic elimination is independent of presynaptic elimination, but presynaptic elimination depends on postsynaptic elimination; in other words, postsynaptic elimination triggers presynaptic elimination. This hypothesis is supported by a previous report that local ecdysone treatment of the hawkmoth, Manduca sexta, to induce local muscle degeneration results in loss of synaptic contacts in the treated region, whereas neighboring NMJ synapses remain intact (Hegstrom and Truman, 1996). Our data are also consistent with mounting evidence from mammalian studies supporting a major role for the postsynaptic side in synapse elimination (Balice-Gordon and Lichtman, 1994; Ichise et al., 2000; McCann et al., 2007; Kano and Hashimoto, 2009; Lorenzetto et al., 2009). However, the downstream targets of the ubiquitination, apoptosis, or ecdysone pathways in the postsynaptic muscles that are crucial for initiating presynaptic dismantling are currently unknown. It will be of great interest to identify these targets.

\section{References}

Allen MJ, Shan X, Caruccio P, Froggett SJ, Moffat KG, Murphey RK (1999) Targeted expression of truncated glued disrupts giant fiber synapse formation in Drosophila. J Neurosci 19:9374-9384.

Awasaki T, Ito K (2004) Engulfing action of glial cells is required for programmed axon pruning during Drosophila metamorphosis. Curr Biol 14:668-677.

Awasaki T, Tatsumi R, Takahashi K, Arai K, Nakanishi Y, Ueda R, Ito K (2006) Essential role of the apoptotic cell engulfment genes draper and ced-6 in programmed axon pruning during Drosophila metamorphosis. Neuron 50:855-867.

Bainbridge SP, Bownes M (1981) Staging the metamorphosis of Drosophila melanogaster. J Embryol Exp Morphol 66:57-80.

Balice-Gordon RJ, Lichtman JW (1994) Long-term synapse loss induced by focal blockade of postsynaptic receptors. Nature 372:519-524.

Bishop DL, Misgeld T, Walsh MK, Gan WB, Lichtman JW (2004) Axon branch removal at developing synapses by axosome shedding. Neuron 44:651-661.

Brand AH, Perrimon N (1993) Targeted gene expression as a means of altering cell fates and generating dominant phenotypes. Development 118:401-415.

Budnik V, Koh YH, Guan B, Hartmann B, Hough C, Woods D, Gorczyca M (1996) Regulation of synapse structure and function by the Drosophila tumor suppressor gene dlg. Neuron 17:627-640.

Chen K, Featherstone DE (2005) Discs-large (DLG) is clustered by presynaptic innervation and regulates postsynaptic glutamate receptor subunit composition in Drosophila. BMC Biol 3:1.

Cherbas L, Hu X, Zhimulev I, Belyaeva E, Cherbas P (2003) EcR isoforms in Drosophila: testing tissue-specific requirements by targeted blockade and rescue. Development 130:271-284. 
Collins CA, DiAntonio A (2007) Synaptic development: insights from Drosophila. Curr Opin Neurobiol 17:35-42.

Collins CA, Wairkar YP, Johnson SL, DiAntonio A (2006) Highwire restrains synaptic growth by attenuating a MAP kinase signal. Neuron 51:57-69.

Colman H, Nabekura J, Lichtman JW (1997) Alterations in synaptic strength preceding axon withdrawal. Science 275:356-361.

DiAntonio A, Hicke L (2004) Ubiquitin-dependent regulation of the synapse. Annu Rev Neurosci 27:223-246.

DiAntonio A, Haghighi AP, Portman SL, Lee JD, Amaranto AM, Goodman CS (2001) Ubiquitination-dependent mechanisms regulate synaptic growth and function. Nature 412:449-452.

Eaton BA, Fetter RD, Davis GW (2002) Dynactin is necessary for synapse stabilization. Neuron 34:729-741.

Featherstone DE, Rushton E, Rohrbough J, Liebl F, Karr J, Sheng Q, Rodesch CK, Broadie K (2005) An essential Drosophila glutamate receptor subunit that functions in both central neuropil and neuromuscular junction. J Neurosci 25:3199-3208.

Gan WB, Lichtman JW (1998) Synaptic segregation at the developing neuromuscular junction. Science 282:1508-1511.

Goda Y, Davis GW (2003) Mechanisms of synapse assembly and disassembly. Neuron 40:243-264.

Hegstrom CD, Truman JW (1996) Synapse loss and axon retraction in response to local muscle degeneration. J Neurobiol 31:175-188.

Ichise T, Kano M, Hashimoto K, Yanagihara D, Nakao K, Shigemoto R, Katsuki M, Aiba A (2000) mGluR1 in cerebellar Purkinje cells essential for long-term depression, synapse elimination, and motor coordination. Science 288:1832-1835.

Jan LY, Jan YN (1976) Properties of the larval neuromuscular junction in Drosophila melanogaster. J Physiol 262:189-214.

Jin S, Pan L, Liu Z, Wang Q, Xu Z, Zhang YQ (2009) Drosophila tubulinspecific chaperone E functions at neuromuscular synapses and is required for microtubule network formation. Development 136:1571-1581.

Kano M, Hashimoto K (2009) Synapse elimination in the central nervous system. Curr Opin Neurobiol 19:154-161.

Keller-Peck CR, Walsh MK, Gan WB, Feng G, Sanes JR, Lichtman JW (2001) Asynchronous synapse elimination in neonatal motor units: studies using GFP transgenic mice. Neuron 31:381-394.

Kuo CT, Jan LY, Jan YN (2005) Dendrite-specific remodeling of Drosophila sensory neurons requires matrix metalloproteases, ubiquitin-proteasome, and ecdysone signaling. Proc Natl Acad Sci U S A 102:1523015235.

Kuo CT, Zhu S, Younger S, Jan LY, Jan YN (2006) Identification of E2/E3 ubiquitinating enzymes and caspase activity regulating Drosophila sensory neuron dendrite pruning. Neuron 51:283-290.

Lee HH, Jan LY, Jan YN (2009) Drosophila IKK-related kinase Ik2 and Katanin p60-like 1 regulate dendrite pruning of sensory neuron during metamorphosis. Proc Natl Acad Sci U S A 106:6363-6368.

Lee T, Marticke S, Sung C, Robinow S, Luo L (2000) Cell-autonomous requirement of the USP/EcR-B ecdysone receptor for mushroom body neuronal remodeling in Drosophila. Neuron 28:807-818.

Lichtman JW, Colman H (2000) Synapse elimination and indelible memory. Neuron 25:269-278.

Lorenzetto E, Caselli L, Feng G, Yuan W, Nerbonne JM, Sanes JR, Buffelli M (2009) Genetic perturbation of postsynaptic activity regulates synapse elimination in developing cerebellum. Proc Natl Acad Sci US A 106:16475-16480.

Luo L, O'Leary DD (2005) Axon retraction and degeneration in development and disease. Annu Rev Neurosci 28:127-156.

MacDonald JM, Beach MG, Porpiglia E, Sheehan AE, Watts RJ, Freeman MR
(2006) The Drosophila cell corpse engulfment receptor Draper mediates glial clearance of severed axons. Neuron 50:869-881.

Marrus SB, Portman SL, Allen MJ, Moffat KG, DiAntonio A (2004) Differential localization of glutamate receptor subunits at the Drosophila neuromuscular junction. J Neurosci 24:1406-1415.

Martin M, Iyadurai SJ, Gassman A, Gindhart JG Jr, Hays TS, Saxton WM (1999) Cytoplasmic dynein, the dynactin complex, and kinesin are interdependent and essential for fast axonal transport. Mol Biol Cell 10:3717-3728.

McCann CM, Nguyen QT, Santo Neto H, Lichtman JW (2007) Rapid synapse elimination after postsynaptic protein synthesis inhibition in vivo. J Neurosci 27:6064-6067.

Pilling AD, Horiuchi D, Lively CM, Saxton WM (2006) Kinesin-1 and Dynein are the primary motors for fast transport of mitochondria in Drosophila motor axons. Mol Biol Cell 17:2057-2068.

Qin G, Schwarz T, Kittel RJ, Schmid A, Rasse TM, Kappei D, Ponimaskin E, Heckmann M, Sigrist SJ (2005) Four different subunits are essential for expressing the synaptic glutamate receptor at neuromuscular junctions of Drosophila. J Neurosci 25:3209-3218.

Sepp KJ, Schulte J, Auld VJ (2000) Developmental dynamics of peripheral glia in Drosophila melanogaster. Glia 30:122-133.

Stewart BA, Atwood HL, Renger JJ, Wang J, Wu CF (1994) Improved stability of Drosophila larval neuromuscular preparations in haemolymphlike physiological solutions. J Comp Physiol A 175:179-191.

Tissot M, Stocker RF (2000) Metamorphosis in Drosophila and other insects: the fate of neurons throughout the stages. Prog Neurobiol 62:89-111.

Vilinsky I, Stewart BA, Drummond J, Robinson I, Deitcher DL (2002) A Drosophila SNAP-25 null mutant reveals context-dependent redundancy with SNAP-24 in neurotransmission. Genetics 162:259-271.

Wan HI, DiAntonio A, Fetter RD, Bergstrom K, Strauss R, Goodman CS (2000) Highwire regulates synaptic growth in Drosophila. Neuron 26:313-329.

Watts RJ, Hoopfer ED, Luo L (2003) Axon pruning during Drosophila metamorphosis: evidence for local degeneration and requirement of the ubiquitin-proteasome system. Neuron 38:871-885.

Watts RJ, Schuldiner O, Perrino J, Larsen C, Luo L (2004) Glia engulf degenerating axons during developmental axon pruning. Curr Biol 14: 678-684.

Williams DW, Truman JW (2005) Cellular mechanisms of dendrite pruning in Drosophila: insights from in vivo time-lapse of remodeling dendritic arborizing sensory neurons. Development 132:3631-3642.

Williams DW, Kondo S, Krzyzanowska A, Hiromi Y, Truman JW (2006) Local caspase activity directs engulfment of dendrites during pruning. Nat Neurosci 9:1234-1236.

Zhai RG, Bellen HJ (2004) The architecture of the active zone in the presynaptic nerve terminal. Physiology (Bethesda) 19:262-270.

Zhang YQ, Bailey AM, Matthies HJ, Renden RB, Smith MA, Speese SD, Rubin GM, Broadie K (2001) Drosophila fragile X-related gene regulates the MAP1B homolog Futsch to control synaptic structure and function. Cell 107:591-603.

Zhang YQ, Rodesch CK, Broadie K (2002) Living synaptic vesicle marker: synaptotagmin-GFP. Genesis 34:142-145.

Zheng X, Wang J, Haerry TE, Wu AY, Martin J, O'Connor MB, Lee CH, Lee T (2003) TGF-beta signaling activates steroid hormone receptor expression during neuronal remodeling in the Drosophila brain. Cell 112: 303-315.

Zito K, Parnas D, Fetter RD, Isacoff EY, Goodman CS (1999) Watching a synapse grow: noninvasive confocal imaging of synaptic growth in Drosophila. Neuron 22:719-729. 